\title{
Can the MMSE help clinicians predict progression from mild cognitive impairment to dementia? ${ }^{\dagger}$ COMMENTARY ON... COCHRANE CORNER
}

\author{
Alex J. Mitchell
}

\section{SUMMARY}

The Mini-Mental State Examination (MMSE) is the most widely used bedside cognitive test. It has previously been shown to be poor as a casefinding tool for both dementia and mild cognitive impairment (MCI). This month's Cochrane Corner review examines whether the MMSE might be used as a risk prediction tool for later dementia in those with established $\mathrm{MCI}$. From 11 studies of modest quality, it appears that the MMSE alone should not be relied on to predict later deterioration in people with $\mathrm{MCl}$. As this is the case, it is likely that only a combination of predictors would be able to accurately predict progression from $\mathrm{MCl}$ to dementia.

\section{DECLARATION OF INTEREST}

None

There is serious concern about the burden of cognitive impairment in both dementia and pre-dementia. In 2010, more than 35 million people worldwide were estimated to be living with dementia, a number expected to increase rapidly by 2050 (Prince 2013). Despite this, dementia is often not diagnosed promptly (Mitchell 2011). By clinical judgement, clinicians typically identify three out of four cases of dementia, although their diagnoses are often not recorded in medical records (Mitchell 2011). Mild cognitive impairment (MCI) has been conceptualised as a pre-dementia condition that affects $15 \%$ of adults over 75 (Panza 2005). If the diagnosis of dementia is less than perfect, then the diagnosis of MCI is even more haphazard. In the case of MCI, clinicians typically correctly diagnose four out of ten people with MCI but only one in ten of these diagnoses is entered into medical records (Mitchell 2011). MCI is often highlighted because of its potential to progress to dementia. Information on long-term cohorts suggests that annual conversion rates range from $4.2 \%$ for all-cause dementia to $5.8 \%$ for Alzheimer's disease with dementia (Mitchell 2008). However, many individuals diagnosed with MCI do not progress to Alzheimer's disease at an accelerated rate and may even revert to normal. Thus, it is important to better understand who will and will not progress once early signs of cognitive impairment are recognised.

Prediction of later dementia is potentially very useful, but at the same time very complicated. There is a trend to diagnose dementia earlier in the course of the disease, but there is also good reason for caution. False positives could have devastating psychological consequences and false negatives would be similarly unwelcome. The longer the follow-up period and the lower the number of incident cases, the more difficult positive prediction becomes. One attractive option that would be remarkably simple in clinical practice would be to use baseline cognitive scores to model later decline. This has considerable face validity because degree of cognitive impairment is usually indicative of further future decline. Most convenient of all would be if the Mini-Mental State Examination (MMSE) could be used in this capacity because the MMSE is still the most commonly used bedside cognitive test.

\section{The Mini-Mental State Examination (MMSE)}

The MMSE is 40 years old this year (Folstein 1975). It has advocates and detractors, but there is no doubt that it has been successfully disseminated. It is relatively brief and has an internal structure of 20 individual mini-tests covering 11 domains, including orientation, registration, attention or calculation (serial sevens or spelling), recall, naming, repetition, comprehension (verbal and written), writing and construction. In clinical practice the main applications of the MMSE are to help clinicians in the diagnosis of dementia and delirium (Diniz 2007; Mitchell 2014a). Consequently it has been investigated in a casefinding role (that is, confirming a diagnosis)
ROUND THE CORNER
Alex J. Mitchell is a consultant psychiatrist in the Department of Psycho-Oncology, Leicestershire Partnership NHS Trust and the Department of Cancer and Molecular Medicine, University of Leicester. Correspondence Dr Alex J. Mitchell, Department of PsychoOncology, Hadley House, Leicester General Hospital, Leicester LE5 4PW, UK. Email ajm80@le.ac.uk

${ }^{\dagger}$ See p. 362 , this issue 
as well as in a screening role (that is, ruling out healthy individuals and minimising false negatives). Unfortunately, research from our group has shown that it is not successful in a diagnostic role and only modestly successful in a screening role (Mitchell 2009a). Indeed, we recently updated the earlier 2009 diagnostic validity meta-analysis with 45 studies (12 community studies, 7 primary care studies and 26 from specialist settings) (Mitchell 2014a). In specialist settings, our revised meta-analysis showed that the MMSE's sensitivity for diagnosing dementia was $76.9 \%$ and its specificity was $89.9 \%$. More meaningfully, that generated a positive predictive value (PPV) of $71.7 \%$ and a negative predictive value (NPV) of $92.1 \%$ at a prevalence of dementia of $25 \%$. In nonspecialist settings such as primary care, where the prevalence of dementia is typically around $10 \%$ of suspected cases, the PPV was only $40 \%$, but the NPV was $98 \%$.

What about the role of the MMSE in helping clinicians diagnose suspected MCI? In the same publication (Mitchell 2014a) our group found 11 qualifying studies looking at older people with subjective memory complaints who might have MCI presenting to a specialist. Meta-analysis showed an overall sensitivity of $66.9 \%$ and a specificity of $77.6 \%$. Assuming a prevalence of MCI of $25 \%$ then the PPV would be only $50 \%$ and NPV about $88 \%$, suggesting rather inadequate diagnostic ability.

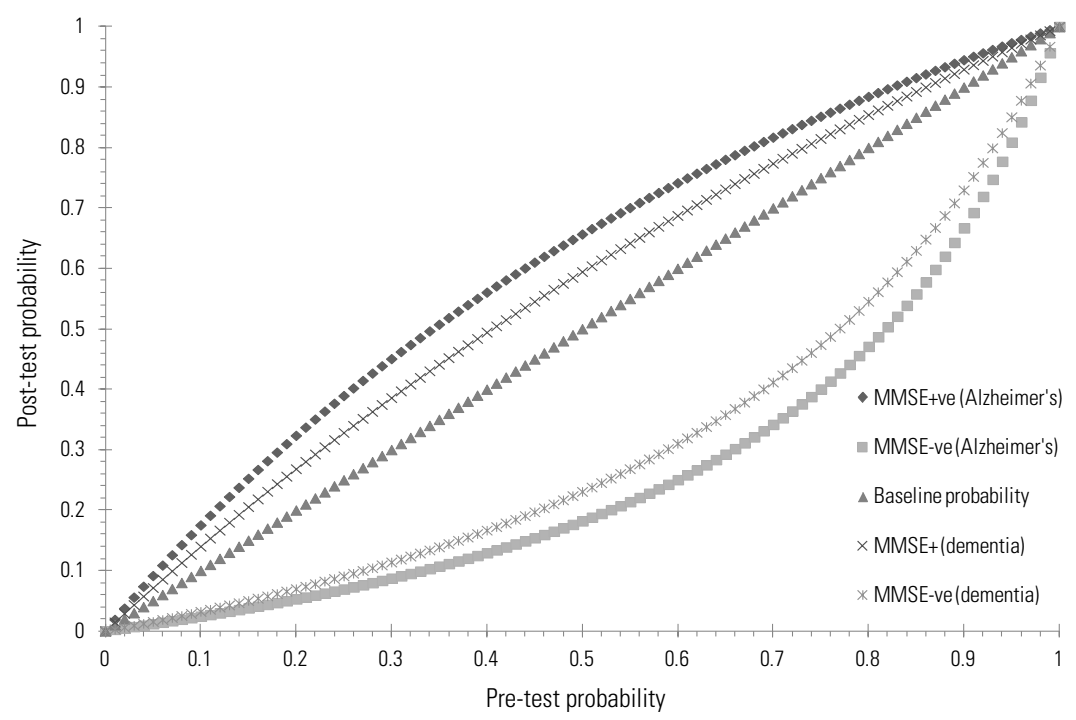

Predictive accuracy of using the Mini-Mental State Examination (MMSE) to spot later dementia or Alzheimer's disease at all possible incidence rates. MMSE+ve, a low (abnormal) MMSE score below the diagnostic threshold; MMSE-ve, a high MMSE score above the diagnostic threshold.

\section{Arevalo-Rodriguez et al's Cochrane review}

Given the diagnostic limitations of the MMSE, it would be surprising, but not impossible, should it prove to be a valuable risk prediction tool. The Cochrane review by Arevalo-Rodriguez and colleagues examined this question of whether the MMSE can be used to predict (rather than diagnose) dementia (Arevalo-Rodriguez 2015). The review included 11 studies, involving 1569 patients with MCI followed for conversion to dementia $(n=4)$ or Alzheimer's disease $(n=8)$ or vascular dementia $(n=1)$. The authors established the diagnosis of MCI using not just conventional and revised Petersen criteria, but also Matthews criteria and the Clinical Dementia Rating (CDR $0.5)$ criteria, which are fairly broad and could be criticised. It should be noted that the QUADAS-2 appraisal tool showed that all 11 studies had a high risk of bias in at least one domain.

Looking in more detail, most of the studies involved samples of older people in memory clinics. Few studies provided descriptive information about social class, years of education, MMSE version used, comorbidities or APOE- $\varepsilon 4$ status or about interventions for MCI given during the follow-up. Also, different diagnostic thresholds $(\leq 21, \leq 26, \leq 28, \leq 29)$ were used to define a positive MMSE result. Importantly, follow-up times ranged from 15 months to 7 years, and over this time the median incidence of all-cause dementia was $36.5 \%$.

Arevalo-Rodriguez et al's results suggest that for conversion from MCI to dementia, the accuracy of baseline MMSE scores showed a wide range of sensitivities (23\% to $76 \%$ ) and specificities (40\% to $94 \%)$. Overall, the authors found that at the median specificity of $88 \%$, the sensitivity was only $40 \%$ for prediction of dementia and $54 \%$ for prediction of Alzheimer's disease. They calculated that in a hypothetical cohort of 100 patients with MCI and a $36.5 \%$ incidence of dementia, there would be 18 false negatives (missed cases) and 8 false positives (patients over-diagnosed).

Further modelling of the accuracy can be generated from a pre-test/post-test Bayesian graph. That in Fig. 1 shows the predictive accuracy of using the MMSE to spot later dementia or Alzheimer's disease calculated from ArevaloRodriguez et al's review. The $y$-axis is the post-test probability, that is, the PPV (if the MMSE score is abnormal) or NPV (if the MMSE score is near normal). The $x$-axis is every possible incidence of future decline. Thus, at a prevalence of $36.5 \%$, the PPV would be $45 \%$ for dementia and $52 \%$ for Alzheimer's disease; conversely, the NPV would be $86 \%$ for dementia and $89 \%$ for Alzheimer's disease. The combined area under the curve would 
be about 0.670 for Alzheimer's disease and 0.627 for dementia, suggesting very modest performance of the MMSE overall.

\section{Conclusions}

Unfortunately, as expected, this Cochrane review suggests that the MMSE when used alone is not a good tool for prediction of future decline in people with MCI. Its most promising role is to reassure those scoring above threshold that a decline to dementia is unlikely. However, even here there is a substantial error rate because about $14 \%$ of those with MCI did decline even with an initially high (that is near normal) MMSE score. Remember that this is in a cohort in which $36.5 \%$ declined over a mean of 3-4 years and therefore a high MMSE score apparently cuts risk of decline by 0.61 of $36.5 \%$ (relative risk reduction). What would be the effect in a primary care setting when the risk of dementia, and hence incidence of dementia, is substantially lower? Assuming that only 10\% decline to dementia, then an above-threshold score on the MMSE would reduce the risk of decline from $10 \%$ to $3 \%$. A below-threshold MMSE score would increase the risk from $10 \%$ to $14 \%$. Thus, perhaps there is a small role for prediction when risk is relatively low, and then only as a temporary reassurance. Overall results are not very encouraging regarding use of the MMSE in this capacity.

\section{Alternative methods to predict dementia}

Assuming that the results are robust and the MMSE is not a good risk prediction tool, are any other methods available that could better predict cognitive decline in MCI? Single-factor models may show a significant association with later dementia, but are unlikely to be accurate enough for clinical reliability. For example, our group recently examined the predictive power of subjective memory complaints in predicting decline to dementia (Mitchell 2014b). Although the annual conversion ratio to dementia was 2.07 (95\% CI 1.76-2.44) in those with v. without subjective memory complaints (SMC), SMC were not useful as a stand-alone diagnostic test because too few people with dementia reliably endorse SMC in the prodromal stages.

To progress further we need to study which factors alone and, more importantly, which factors in combination best predict later dementia. In most cases a combination of markers are more accurate than simple single-factor models (Ewers 2012). For example, Cooper et al (2015) examined reversible dementia risk factors systematically in those who already had MCI. From 30 studies in their review, diabetes and the presence of miscellaneous neuropsychiatric symptoms significantly predicted the conversion of MCI to dementia (Cooper 2015).

However, in order to take the next step multiple factors must be combined mathematically in a risk prediction index or panel. Prediction indexes are slowly appearing in mainstream medical practice, for example in the Framingham cardiovascular risk calculator (D'Agostino 2001). Although this approach has been used to model risk of dementia in cognitively unimpaired older people, only a very small handful of studies have used it in modelling progression of MCI. Further, most of the models have been based on biomarkers such as phosphorylated tau levels in the cerebrospinal fluid (CSF), or neuroimaging or genotyping (Mitchell 2009b; Monge-Argilés 2010; Elias-Sonnenschein 2011; Rodríguez-Rodríguez 2013). Used alone even biomarkers have modest added value in most cohorts and this value is still debated (Mitchell 2010; Cui 2011; Gomar 2011). It is surprising therefore how few studies have tested clinical predictors, or combined clinical predictors with biomarkers (Ferreira 2014).

One promising example is from the Alzheimer's Disease Neuroimaging Initiative 1 (ADNI-1), an ongoing multicentre study initiated in 2003. From the ADNI database, Lee et al (2014) developed a prediction index for the 3-year progression from MCI to dementia using information that can be readily obtained in most clinical settings. Important predictors of progression were female gender, resisting help, becoming upset when separated from caregiver, difficulty shopping alone, forgetting appointments, number of words recalled from a 10-word list, poor orientation and difficulty drawing a clock. These predictors generated a final points score that could range from 0 to 16 . Fourteen per cent of individuals with low scores (0-2 points) converted to probable Alzheimer's disease over 3 years, compared with $91 \%$ of those with high scores (9-16 points). Currently, this is the most promising lead about which combination of clinical factors might help clinicians to predict which patients with early prodromal cognitive impairment might progress to dementia.

\section{References}

Arevalo-Rodriguez I, Smailagic N, Roqué I Figuls M, et al (2015) MiniMental State Examination (MMSE) for the detection of Alzheimer's disease and other dementias in people with mild cognitive impairment (MCI). Cochrane Database of Systematic Reviews, 3: CD010783.

Cooper C, Sommerlad A, Lyketsos CG, et al (2015) Modifiable predictors of dementia in mild cognitive impairment: a systematic review and metaanalysis. American Journal of Psychiatry, 172: 323-34.

Cui Y, Liu B, Luo S, et al (2011) Identification of conversion from mild cognitive impairment to Alzheimer's disease using multivariate predictors. PLoS One, 6: e21896. 
D'Agostino Sr RB, Grundy S, Sullivan LM, et al (2001) Validation of the Framingham coronary heart disease prediction scores: results of a multiple ethnic groups investigation. JAMA, 286: 180-7.

Diniz BSO, Yassuda MS, Nunes PV, et al (2007) Mini-mental State Examination performance in mild cognitive impairment subtypes. International Psychogeriatrics, 19: 647-56.

Elias-Sonnenschein LS, Viechtbauer W, Ramakers IH, et al (2011) Predictive value of APOE- $\varepsilon 4$ allele for progression from MCI to AD-type dementia: a meta-analysis. Journal of Neurology, Neurosurgery, and Psychiatry, 82: 1149-56.

Ewers M, Walsh C, Trojanowski JQ, et al (2012) Prediction of conversion from mild cognitive impairment to Alzheimer's disease dementia based upon biomarkers and neuropsychological test performance. Neurobiology of Aging, 33: 1203-14.

Ferreira D, Rivero-Santana A, Perestelo-Pérez L, et al (2014) Improving CSF biomarkers' performance for predicting progression from mild cognitive impairment to Alzheimer's disease by considering different confounding factors: a meta-analysis. Frontiers in Aging Neuroscience, 6: 287.

Folstein MF, Folstein SE, McHugh PR (1975) 'Mini-mental state': a practical method for grading the cognitive state of patients for the clinician. Journal of Psychiatric Research, 12: 189-98.

Gomar JJ, Bobes-Bascaran MT, Conejero-Goldberg C, et al (2011) Utility of combinations of biomarkers, cognitive markers, and risk factors to predict conversion from mild cognitive impairment to Alzheimer disease in patients in the Alzheimer's disease neuroimaging initiative. Archives of General Psychiatry, 68: 961-9.

Lee SJ, Ritchie CS, Yaffe K, et al (2014) A clinical index to predict progression from mild cognitive impairment to dementia due to Alzheimer's disease. PloS One, 9: e113535.

Mitchell AJ, Shiri-Feshki M (2008) Temporal trends in the long term risk of progression of mild cognitive impairment: a pooled analysis. Journal of Neurology, Neurosurgery, and Psychiatry, 79: 1386-91.
Mitchell AJ (2009a) A meta-analysis of the accuracy of the Mini-Mental State Examination in the detection of dementia and mild cognitive impairment. Journal of Psychiatric Research, 43: 411-31.

Mitchell AJ (2009b) CSF phosphorylated tau in the diagnosis and prognosis of mild cognitive impairment and Alzheimer's disease: a meta-analysis of 51 studies. Journal of Neurology, Neurosurgery, and Psychiatry, 80: 966-75.

Mitchell AJ, Monge-Argilés JA, Sánchez-Paya J (2010) Do CSF biomarkers help clinicians predict the progression of mild cognitive impairment to dementia? Practical Neurology, 10: 202-7.

Mitchell AJ, Meader N, Pentzek M (2011) Clinical recognition of dementia and cognitive impairment in primary care: a meta-analysis of physician accuracy. Acta Psychiatrica Scandinavica, 124: 165-83.

Mitchell AJ (2014a) The Mini-Mental State Examination (MMSE): an update on its diagnostic validity for cognitive disorders. In Cognitive Screening Instruments: A Practical Approach (ed AJ Larner): 15-46. Springer.

Mitchell AJ, Beaumont H, Ferguson D, et al (2014b) Risk of dementia and mild cognitive impairment in older people with subjective memory complaints: meta-analysis. Acta Psychiatrica Scandinavica, 130: 439-51.

Monge-Argilés JA, Sánchez-Payá J, Muñoz-Ruiz C, et al (2010) Biomarkers in the cerebrospinal fluid of patients with mild cognitive impairment: a meta-analysis of their predictive capacity for the diagnosis of Alzheimer's disease. Revue Neurologique, 50: 193-200

Panza F, D'Introno A, Colacicco AM, et al (2005) Current epidemiology of mild cognitive impairment and other predementia syndromes. American Journal of Geriatric Psychiatry, 13: 633-44.

Prince M, Bryce R, Albanese E, et al (2013) The global prevalence of dementia: a systematic review and metaanalysis. Alzheimer's \& Dementia, 9: 63-75.

Rodríguez-Rodríquez E, Sánchez-Juan P, Vázquez-Higuera JL, et al (2013) Genetic risk score predicting accelerated progression from mild cognitive impairment to Alzheimer's disease. Journal of Neural Transmission, 120: $807-12$ 